\title{
Sexually Selected Egg Coloration in Spotless Starlings
}

\author{
Juan J. Soler, ${ }^{\star}$ Carlos Navarro, ${ }^{\dagger}$ Tomás Pérez Contreras, ${ }^{\ddagger}$ Jesús M. Avilés, ${ }^{\S}$ and José J. Cuervo
}

Departamento de Ecología Funcional y Evolutiva, Estación Experimental de Zonas Áridas (Consejo Superior de Investigaciones Científicas), E-04001 Almería, Spain

Submitted March 30, 2007; Accepted September 25, 2007; Electronically published December 7, 2007

Online enhancements: color versions of figures 1 and 2.

ABSTRACT: It has been recently proposed that the blue-green coloration in eggs of many avian species may constitute a sexually selected female signal. Blue-green color intensity would reflect the physiological condition of females, and hence it might also affect the allocation of male parental care. In this study, we use three different experimental approaches to explore the importance of sexual selection on blue-green egg coloration of spotless starling (Sturnus unicolor) eggs. First, experimental deterioration of female body condition (by means of wing feather removal) negatively affected the intensity of blue-green egg coloration. Second, blue-green color intensity of artificial model eggs had a significant positive influence on paternal feeding effort. Finally, we found a negative relationship between the effect of experimental food supply on nestling immunocompetence and the intensity of blue-green coloration of eggs, suggesting that egg color predicts nutritional conditions that nestlings will experience during development. All these results taken together strongly support a role of sexual selection in the blue-green coloration of spotless starling eggs.

Keywords: blue eggs, differential allocation hypothesis, egg coloration, feeding effort, sexual selection, spotless starling.

The evolution of egg coloration, its intra- and interspecific variability, and its possible functionality are questions that have attracted evolutionary ecologists for many years, giving rise to different hypotheses (Underwood and Sealy

* Corresponding author; e-mail: jsoler@eeza.csic.es.

† E-mail: cnavarro@eeza.csic.es.

* E-mail: tomaspc@eeza.csic.es.

s E-mail: javiles@eeza.csic.es.

" E-mail: jcuervo@eeza.csic.es.

Am. Nat. 2008. Vol. 171, pp. 183-194. (C) 2007 by The University of Chicago. 0003-0147/2008/17102-42509\$15.00. All rights reserved.

DOI: $10.1086 / 524958$
2002; Kilner 2006). The most traditional functional hypothesis is related to avoidance of predators, and in accordance, species that nest directly on the ground (i.e., with high risk of predation) lay more cryptic eggs (e.g., Solis and de Lope 1995). Another functional hypothesis is related to brood parasitism. In a brood parasitic scenario, it would be of selective advantage for hosts to lay eggs that differ from those laid by parasitic individuals (Davies and Brooke 1988; Petrie and Møller 1991; Soler and Møller 1996; Stokke et al. 2002). A third hypothesis is based on the mechanical and thermal properties of protoporphirins and posits the functionality of the reddish eggshell spots as affecting eggshell strength (Gosler et al. 2005). Finally, trying to explain the typical blue-green background coloration of eggs of many species, which until recently was considered a mystery (Underwood and Sealy 2002), Moreno and Osorno (2003) proposed that the blue and green egg colors may constitute a signal of females that reflects their physiological condition. Consequently, high bluegreen color intensity would induce in males a high allocation of parental effort, according to the differential allocation hypothesis (Burley 1986; Sheldon 2000).

This last hypothesis is based on the fact that the pigment responsible for blue-green coloration in the eggshell (Kennedy and Vevers 1976) is a potent antioxidant (Stocker et al. 1987). Because females are under high levels of oxidative stress while laying eggs (e.g., Von Schantz et al. 1999; Alonso-Alvarez et al. 2004), only those females with a high antioxidant capacity would be able to exaggerate the use of biliverdin as an eggshell pigment (Moreno and Osorno 2003). The hypothesis of blue-green egg coloration being a sexually selected signal of females has recently received comparative (Soler et al. 2005), empirical, and some experimental support. For instance, body condition and immunocompetence of females, and also those of nestlings, are related to egg color in some species (Moreno et al. 2005; Siefferman et al. 2006; Krist and Grim 2007). Moreover, blue-green color intensity of eggs of pied flycatchers (Ficedula hypoleuca) both positively correlates with biliverdin contents in the eggshell and reflects the nutritional condition of females at laying (Moreno et al. 2006a). Eggs of this species, and also those of the closely related collared flycatcher (Ficedula albicollis), become paler in the course 
of the laying sequence, suggesting a pigment limitation (Moreno et al. 2005; Krist and Grim 2007). Moreover, blue-green color intensity was positively related to immunoglobulin concentration in the yolk and in the blood of females and offspring (Morales et al. 2006). Finally, although there is correlative evidence of the hypothetical positive effects of egg coloration on paternal effort (i.e., provisioning rate; Moreno et al. 2004), cross-fostering experiments of clutches of collared and pied flycatcher produced diverse results. While in pied flycatchers the bluegreen color intensity of experimental eggs, but not that of original eggs, was positively related to the proportion of male provisioning visits (Moreno et al. 2006b), egg color of collared flycatchers did not affect male feeding rates (Krist and Grim 2007). Therefore, although the informational content of blue egg color is not in doubt, because of the association with variables related to female and offspring quality, cross-fostering experiments with eggs do not offer full support for the crucial prediction of the hypothesis that males allocate effort in relation to blue color intensity.

The hypothesis also applies to an interspecific context. Indeed, it has been shown that European passerines that lay eggs of higher intensity of blue-green coloration experience longer nestling periods after controlling for body mass, a measure of parental investment, and higher degree of polygyny (Soler et al. 2005). Moreover, hole-nesting species lay eggs of higher blue-green color intensity than open nesters, which has been suggested to reflect different selection pressures (e.g., risk of parasitism, light conditions) affecting species with the two kinds of nests (Soler et al. 2005).

In this study, we have used three different experimental approaches to explore three main predictions of the hypothesis that blue-green egg color intensity is a sexually selected signal of females (sexual selection hypothesis $[\mathrm{SSH}]$ ) in a population of spotless starlings (Sturnus unicolor). Starlings are strict hole-nesting species, and experimental evidence has shown that they have the visual potential to detect subtle differences in egg coloration within their nests (Aviles et al. 2006a). These predictions are that egg color (1) indicates female condition at laying, (2) affects parental investment of males, and therefore (3) predicts nutritional conditions that nestlings experience during growth (as long as females do not change their feeding effort in response to that of their mates). Briefly, the first prediction was explored by experimental manipulation of female condition (i.e., cutting off some wing feathers of females several weeks before laying) and analyzing the effect on body mass loss and egg coloration. To test the second prediction, during laying we daily exchanged natural eggs with artificial eggs made of plaster of Paris and resembling the shape and color of natural starling eggs, and we explored its effect on male provisioning rates. Finally, we indirectly tested the third prediction by food supplementing some nestlings in the nest while leaving the remaining siblings as controls. Because the effect of our experimental food supply would be larger in nests of poor nutritional conditions (i.e., low parental investment), we used within-nest differences between food-supplemented and control nestlings in nutritional conditioned variables (e.g., immune response) as an index of nutritional conditions experienced by nestlings during development (for a similar approach, see De Neve et al. 2004c). We therefore predicted larger differences in nutritional conditions in those nests with less blue-green egg coloration.

\section{Material and Methods}

The study was carried out in Guadix $\left(37^{\circ} 18^{\prime} \mathrm{N}, 3^{\circ} 11^{\prime} \mathrm{W}\right)$, southeastern Spain, during the breeding seasons of 2005 and 2006 in nest boxes recently (2005-2006) installed close to or within colonies of spotless starlings already established in old buildings in the area. Adults were captured inside nest boxes during nest building, then sexed and marked with a metallic-numbered ring and a unique combination of colored plastic rings.

The spotless starling is a sexually dimorphic (Veiga et al. 2001) species that lays nonspotted blue-greenish eggs (Cramp 1998) with a small peak at the ultraviolet wavelength (fig. 1). Females lay one egg per day, mainly during the morning, and start incubation before clutch completion, which determines asynchronous hatching (see Cramp 1998). In our population, clutch size is typically four to five eggs (data from 2005 and 2006 combined; $N=159$; mean $=4.55 ; \mathrm{SD}=0.97$; range $=2-7$ ), and except for a few exceptions, incubation is a female task (C. Navarro, T. P. Contreras, J. M. Avilés, and J. J. Soler, unpublished data). Nestlings are fed mainly insects (Motis et al. 1997) by both males and females (Cramp 1998; Veiga et al. 2002). Male feeding visits occur mainly during the first half of the nestling period, when feeding rates of males are usually higher than those of females (see "Results").

\section{Female Condition Manipulation}

About 1 month before egg laying (mean $=32.0$ days, $\mathrm{SD}=17.8, N=32$ ), we captured individuals that slept inside nest boxes, or we visited them during the morning. We manipulated the wing area of female starlings following the protocol established by Møller et al. (1995). Briefly, during the field seasons of 2005 and 2006, all captured females were weighed, measured, and randomly assigned to one of the three following treatments: (1) primaries 4 and 5 were removed by cutting off the feathers at the base (manipulation; $N=33$ ); (2) the outermost $1 \mathrm{~mm}$ of pri- 


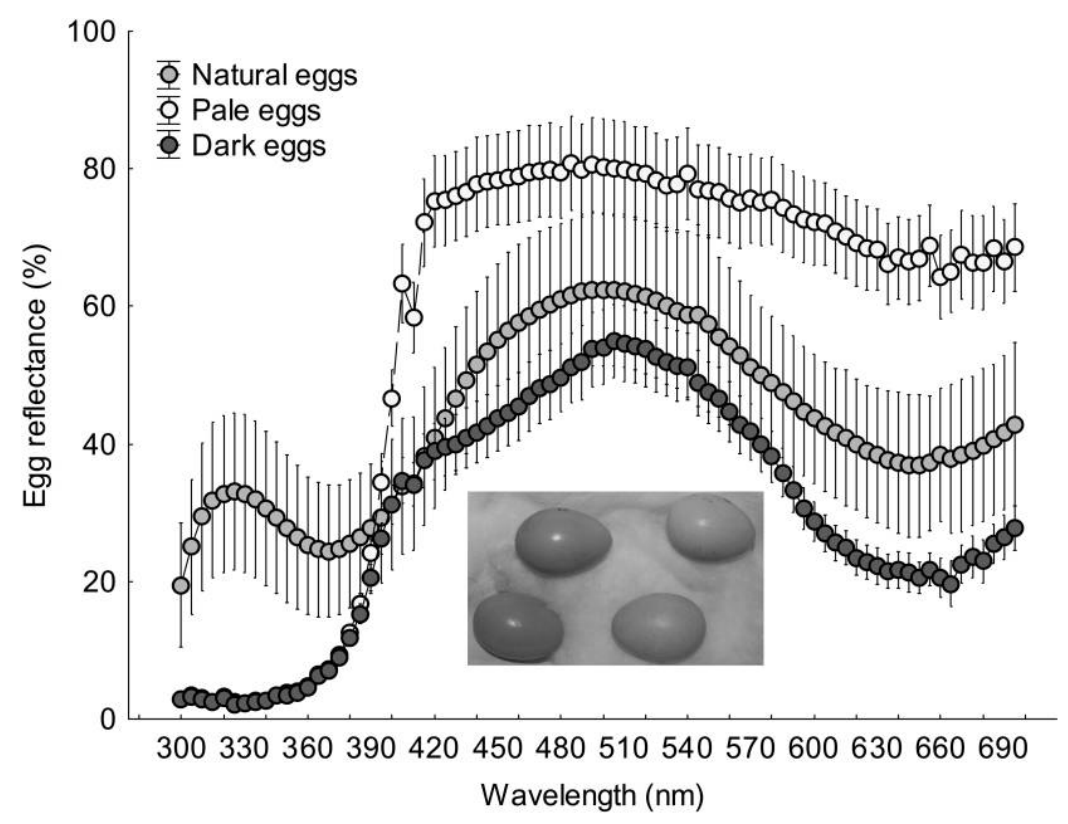

Figure 1: Mean reflectance $( \pm S D)$ spectra of natural spotless starling (Sturnus unicolor) eggs. Reflectance curves for experimental model eggs (pale and dark) are also shown. Experimental pale (right) and dark (left) eggs used in the experiment are shown in the lower part of the picture, while natural eggs of similar coloration are shown in the upper part. A color version of this figure is available in the online edition of the American Naturalist.

maries 4 and 5 was removed (sham manipulation; $N=$ $21)$; and (3) the female was captured and the wings handled as in treatments 1 and 2 (control; $N=28$ ). This experiment reduced wing area of experimental females about $5 \%$, and thus wing loading increased at the same rate. The experiment did not affect probability of reproduction since the percentage of females for which we detected reproduction did not vary for different treatments (experimental, 42.9\%; sham manipulated, $42.9 \%$; control, $42.4 \%$; generalized linear model with binomial error and logistic function, Wald $=0.11, P=.95$ ). Thus, after removing nests from two control and one experimental females for which we lost egg color measurements, we collected information on egg coloration for 11 experimental, 9 sham-manipulated, and 12 control females. The effect of capture and handling of females on blue-green color intensity of their eggs was assessed by comparing egg color of females captured before egg laying with that of seven females that were trapped after egg laying. In 2006, about 1 or 2 weeks after the first capture but before egg laying, we recaptured and weighed 13 females and used differences between first and second weights to validate our experiment (i.e., variation in body mass from first to second capture should be larger for experimental than for control females). We did not find between-year differences in egg coloration $(F=0.08, \mathrm{df}=1,26, P=.78)$ or in the effect of our experiment on egg color (interaction between year and experimental treatment; $F=0.04, \mathrm{df}=2,26, P=$ .96). Thus, we pooled data from both years.

\section{Egg Color Manipulation and Estimation of Parental Feeding Effort}

During the nest-building period of 2006, we visited nest boxes daily until clutch completion. Once the first egg was detected and every day until clutch completion, each new laid egg was exchanged with either a pale $(N=11$ nests $)$ or a dark ( $N=11$ nests) blue-green model egg (see fig. 2). Original clutch size of nests with pale (mean \pm $\mathrm{SD}=4.8 \pm 1.3$ eggs) and dark (mean $\pm \mathrm{SD}=4.6 \pm$ 0.7 eggs) artificial eggs did not differ significantly $(t=$ $0.40, \mathrm{df}=20, P=.69)$. Artificial model eggs were made of plaster of Paris using latex molds from natural spotless starling eggs. Later, model eggs were painted with acrylic paints to produce colors similar to those of the least and most pigmented eggs in our population. All pale and dark artificial eggs were colored with the same two kinds of paint (Natural Color System, Scandinavian Colour Institute; dark eggs: 2030-B90G; pale eggs: $10 \%$ of dark eggs' paint and $90 \%$ of pure white). Artificial colored eggs, however, did not reflect in the UV part of the spectrum (fig. 1). Thus, although blue-green coloration (i.e., chroma) of 


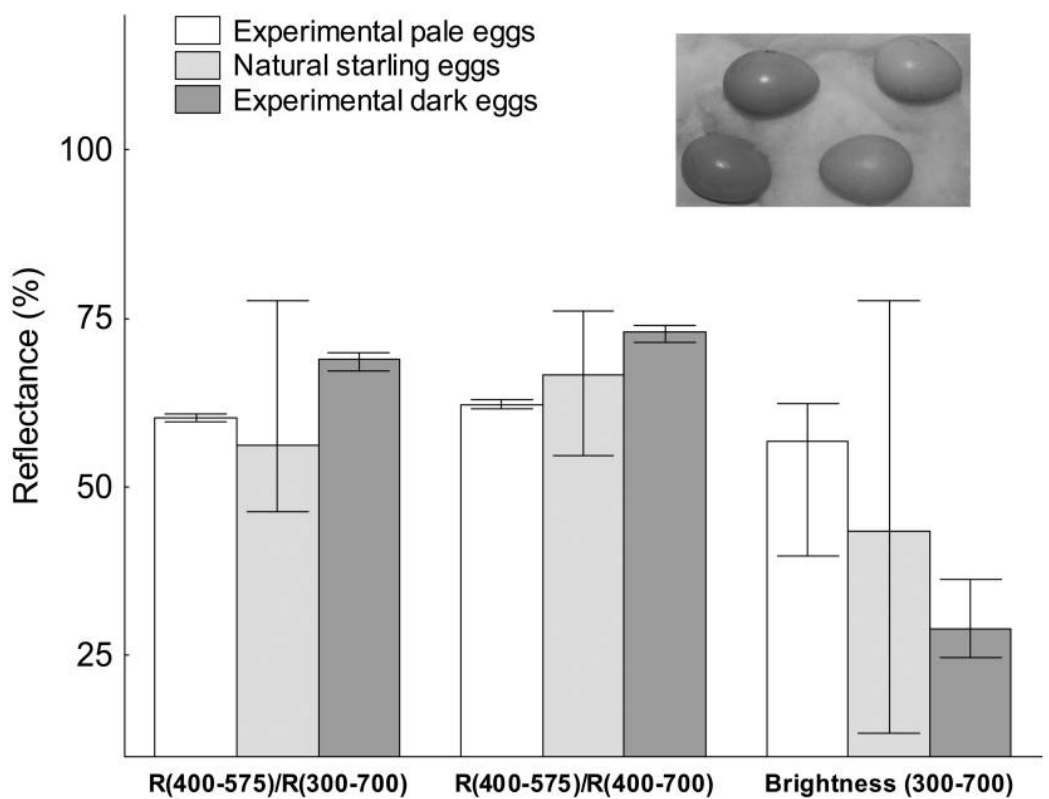

Figure 2: Mean, maximum, and minimum percentage of reflectance for blue-green (400-575 nm) colors relative to total (300-700 nm) or human visual $(400-700 \mathrm{~nm})$ spectrum of experimental (pale eggs, $N=20$; dark eggs, $N=20)$ and natural $(N=814)$ spotless starling eggs. Mean values of total reflectance (i.e., brightness) for natural and experimental eggs are also shown. Experimental pale (right) and dark (left) eggs used in the experiment are shown in the lower part of the picture, while natural eggs of similar coloration are shown in the upper part. A color version of this figure is available in the online edition of the American Naturalist.

spotless starling eggs was intermediate between artificial pale and dark eggs for the visible part of the spectrum (400-700 nm), it showed smaller values than those estimated for artificial eggs when the complete reflectance spectrum (300-700 nm) was considered (fig. 2). In any case, coloration of experimental eggs was within the natural variation, even when UV reflectance was taken into account (see fig. 2). Model eggs colored with nonreflecting UV paints have provided biologically meaningful results in contexts in which visual detection of a receiver bird in the nest was implicated (e.g., brood parasitism; see Davies 2000). Moreover, plaster of Paris model eggs can be easily painted and, on average, do not differ from natural eggs in weight (e.g., Soler and Soler 2000).

We assume that males detected mainly experimental model eggs in their nests because natural eggs from experimental nests were in their original nests for only a few hours. Additionally, to be sure that provisioning effort in experimental nests was within the natural range of variation, some other nests $(N=29)$ were kept as controls for comparisons with experimental nests.

Two weeks after the first egg was laid, which corresponds to the typical incubation period for the species (Cramp 1998), experimental eggs were replaced by two to three hatchlings from randomly selected spotless starling nests that were not used in the experiment. Consequently, nest- lings in these experimental nests were never related to the adults taking care of them. It should be noted that brood size in these experimental nests (two to three nestlings) was smaller than original clutch size (usually four or five eggs; see above).

Provisioning behavior of adults was observed three times in the course of the nestling period, specifically when nestlings were 4 (mean $\pm \mathrm{SD}=4.0 \pm 1.5$ days, $N=$ 48 [22 experimental nests]), 9 (mean $\pm \mathrm{SD}=8.6 \pm 2.1$ days, $\quad N=43 \quad[20$ experimental nests]), and 15 (mean $\pm \mathrm{SD}=14.8 \pm 1.6$ days, $N=37$ [19 experimental nests]) days old. All observations were performed during the morning. Parental visits were recorded by direct observation of the nests with a telescope (from a blind or a car) during periods of approximately $1 \mathrm{~h}$ (mean \pm $\mathrm{SD}=59.8 \pm 3.37 \mathrm{~min}, N=127)$. When the number of nests to be observed was large, some nests (chosen blindly with regard to the experimental treatment) were recorded with a video camera placed several meters away from the nest box. Observations or recordings with no detection of parental feeding visits were not included in the analyses. Provisioning rates have been corrected to $1-\mathrm{h}$ periods by dividing the number of visits by the number of minutes recorded and multiplying by 60 . For each visit, we identified the sex and also whether adults carried food for feeding the offspring (i.e., feeding visits). 
Table 1: Mean (SE) values and results from repeated-measures ANOVA

\begin{tabular}{lccrc}
\hline & Experimental nestlings & Control nestlings & \multicolumn{1}{c}{$F$} & $P$ \\
\hline PHA response $(\mathrm{mm})$ & $.65(.03)$ & $.56(.03)$ & 6.66 & .001 \\
Body mass $(\mathrm{g})$ & $77.5(1.2)$ & $78.5(1.1)$ & .46 & .50 \\
Tarsus length $(\mathrm{mm})$ & $29.7(.2)$ & $30.0(.2)$ & 2.11 & .16 \\
Body condition & $.32(1.1)$ & $.24(1.1)$ & .01 & .94 \\
\hline
\end{tabular}

Note: Mean (SE) values are for T-cell-mediated immune response (PHA response), body mass, tarsus length, and body condition in control and experimental food-supplemented nestlings. For ANOVA, treatment was the repeated measure. For $F$ values, $\mathrm{df}=1,33$.

\section{Manipulation of Nestlings' Nutritional Condition}

During the breeding season of 2005, 3 days after the first nestling hatched, each hatchling was weighed, marked with a waterproof pen on the tarsus, and ranked according to body mass within each nest. We manipulated the nutritional condition of nestlings by means of food supplementation of some nestlings within the nest. The heaviest nestling was randomly assigned either to be food supplemented or to receive water as a control. Feeding treatment was alternated through the mass-based rank within the nest. The food treatment consisted of $0.2 \mathrm{~mL}$ of calorierich pasta loaded with essential micronutrients (minerals, vitamins, and amino acids; 5 calories per gram; NutriCalorías, Schering-Plough Animal Health). Subsequently, we revisited the nests every second day (five times during the nestling period), recolored the tarsi of all nestlings, and fed the nestlings assigned to control and experimental treatments during the first visit.

Nestlings were ringed about 4 days before fledging, that is, when they were 13-14 days old. At that moment, they were also weighed (with a Pesola spring balance to the nearest $0.5 \mathrm{~g}$ ) and measured (the tarsus with a digital caliper to the nearest $0.01 \mathrm{~mm}$, and wing length with a ruler to the nearest $0.1 \mathrm{~cm}$ ). An index of body condition was estimated by regressing body mass on tarsus length and calculating the residuals (Schulte-Hostedde et al. 2005). Moreover, as a variable related to nutritional condition of nestlings, we also used values of skin swelling elicited by injection of the mitogen phytohemagglutinin (PHA; Sigma Chemical). It is well known that this response reflects nutritional conditions that nestlings have experienced during development (e.g., Alonso-Alvarez and Tella 2001). It is also commonly used in evolutionary ecology to estimate T-cell-mediated immunity (Kennedy and Nager 2006), although it also reflects other components of the immune system, such as major histocompatibility complex molecules (e.g., Goto et al. 1978; Martin et al. 2006). Fledglings were injected subcutaneously in the right wing web with $0.2 \mathrm{mg}$ of PHA dissolved in $0.04 \mathrm{~mL}$ of physiological saline solution (Bausch and Lomb). The left wing web was injected with $0.04 \mathrm{~mL}$ of physiological saline solution. We measured the thickness of each wing web at the injection site (with a Mitutoyo model ID-CI012 BS, to the nearest $0.01 \mathrm{~mm}$ ) before and $24 \mathrm{~h}$ after the injection and estimated immune response as the change in thickness of the right wing web (PHA injection) minus the change in thickness of the left wing web. We repeated measurements of each wing web three times, and the mean was used in subsequent analyses.

Repeated-measures ANOVA with treatment (food supplemented vs. control) as a repeated measure revealed that food supplementation had a significant effect on nestling PHA response but not on tarsus length, body mass, or body condition index (table 1). Thus, within-nest differences between food-supplemented and control nestlings in PHA response were used as an index related to nutritional conditions that nestlings experienced during development (hereafter, nutritional condition of nestlings; for a similar approach, see De Neve et al. 2004c).

From the 36 nests with egg color information and experimental food supply, six were depredated before fledging, and only control or experimental nestlings fledged in three and two nests, respectively. Therefore, for 25 nests, we have both egg color information and the effect of our food supply experiment for control and experimental nestlings.

\section{Egg Color Measurements}

Egg coloration was measured within 2 days after the end of laying. To standardize ambient light conditions, all the measurements were taken inside a portable blind with opaque walls set up close to the nests. To prevent nest desertion by parents, nest box entrances were always blocked while measuring reflectance spectra. Eggs were returned to their nest within 15 min of removal, and subsequent visits to these nests confirmed that none of them was deserted. Color was always measured on five randomly selected areas of the surface of the egg along the long egg axis (Aviles et al. 2006a, 2006b). Reflectance spectra (300$700 \mathrm{~nm}$ ) were recorded using an Ocean Optics S2000 spectrometer connected to a deuterium-halogen light (D2-W, mini) by a coaxial reflectance probe (QR-400-7-UV-vis) and the OOIBase 32 operating software (Ocean Optics, 
Dunedin, FL). Reflectances were always measured with the probe placed at a constant distance and reaching the egg at $45^{\circ}$. Measurements were relative and referred to a standard white reference (WS-2) and to the dark, both calibrated before the measurement of each clutch.

As a variable indicating intensity of blue-green coloration, we used the proportion of reflectance in the bluegreen region of the spectrum $(\mathrm{R}[400-575] / \mathrm{R}[300-700]$; Siefferman et al. 2006), the region where biliverdin shows the lowest absorbance (Falchuk et al. 2002) and where reflectance of starling eggs reaches its maximum (fig. 1). Estimates of this variable at five different areas of the eggshell showed significant repeatabilities (data from 2005; $r=0.87, F=6.7, \mathrm{df}=205,824, P<.0001)$. Betweennest variation in this variable was significantly larger than within-nest variation $(r=0.85, F=17.4$, df $=48,158$, $P<.0001)$, and thus mean values were used for each clutch.

\section{Statistical Methods}

All variables were approximately normally distributed (Kolmogorov-Smirnov test for continuous variables, $P>$ .2 ), and, consequently, parametric statistics were used to analyze the data. To test the effects of female wing feather removal on body mass loss and egg color, we used regression analyses, one-way ANOVAs, and repeatedmeasures ANOVAs. To test the effect of our egg color experiment (i.e., dark vs. pale blue-greenish eggs) on male provisioning rates, we used a general linear model with experimental treatment (i.e., pale or dark eggs) and nestling development stage (i.e., first, 4 days; second, 9 days; third, 15 days after hatching) as fixed effects. To account for the nonindependence of observations at the same nest but at different nestling ages, nest identity, nested within experimental treatment, was included in the model as a random factor. Moreover, since male provisioning rate may depend on that of females but also on brood size, hatching date, and nestling age (i.e., variation from the typical development stage at which observations were performed), all these variables were included in a first model as covariates. After testing the model, including all mentioned factors and covariables as well as the interaction between fixed factors (hereafter, full model), we systematically removed the variable associated with the largest $P$ value and ran the model again until we reached a model with all factors and covariables associated with two-tailed $P$ values smaller than 0.1 (hereafter, final model). Residuals from statistical models approximately followed a normal distribution (Kolmogorov-Smirnov test, $P>.2$ ), and variances in provisioning rate of different groups were homogeneous (Levene's test for homogeneity of variances, $F=1.09, \mathrm{df}=5,54, P=.37$ ), validating the use of para- metric tests. Since some nests involved in the egg color experiment were predated during the nestling period, sample sizes of parental provisioning behavior differ between nestling development stages.

Finally, to test the effect of our food supply experiment on the hypothetical relationship between egg coloration and nestling phenotype, we explored the relationship between these variables for control and experimental nestlings in a repeated-measures ANCOVA (i.e., treatment as a within factor and variables related to egg coloration as covariables). Regression analyses were used to test for the relationship between effect sizes within nests in our food supply experiment. All the analyses were performed with the STATISTICA 6 software (StatSoft 2001), and, conservatively, we used two-tailed $P$ values.

\section{Results}

\section{Removal of Wing Feathers and Egg Color}

Blue-green egg color intensity of control females captured before reproduction $(\mathrm{R}[400-575] / \mathrm{R}[300-700]$, mean $=$ $55.32, \mathrm{SD}=2.47, N=12$ ) did not differ significantly from egg color of females that were captured after laying $(\mathrm{R}[400-575] / \mathrm{R}[300-700], \quad$ mean $=55.87, \quad \mathrm{SD}=2.89$, $N=7$ ), suggesting that female capture and handling did not affect blue-green color intensity of the eggs. The comparison of body mass variation of females that were captured twice before egg laying in relation to experimental treatment revealed that the experimental removal of wing feathers had a detrimental effect on female body condition (repeated-measures ANOVA, body mass of females at different capture dates as within effect, and experimental treatment as between effect; interaction term: $F=8.56$, $\mathrm{df}=1,11, P=.014)$. While body mass of experimental females (i.e., with wing feathers removed) decreased between first and second capture (first capture: mean body mass $\pm \mathrm{SD}=84.4 \pm 4.7 \mathrm{~g}, N=6$; second capture: mean body mass $\pm \mathrm{SD}=80.7 \pm 3.1 \mathrm{~g}, N=6$ ), that of control females tended to increased in the same period (first capture: mean body mass $\pm \mathrm{SD}=81.1 \pm 3.5 \mathrm{~g}, N=7$; second capture: mean body mass $\pm \mathrm{SD}=86.6 \pm 8.0 \mathrm{~g}$, $N=7$ ). This effect was independent of time variation between captures (time between first and second capture: mean $\pm \mathrm{SD}=8.6 \pm 3.0$ days) because when this information was included as a covariate in the previous model, the interaction term between body mass change and experimental treatment was still statistically significant ( $F=6.53, \mathrm{df}=1,10, P=.029)$. Thus, the experimental removal of wing feathers effectively provoked a reduced body condition in experimental females.

Control and sham-manipulated females laid eggs that were more blue-green than those laid by experimental fe- 


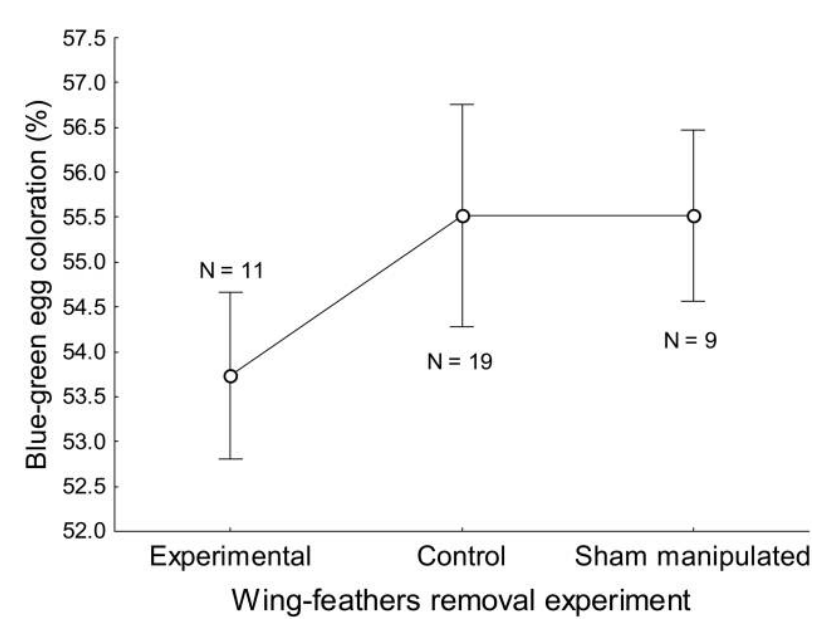

Figure 3: Weighted means ( $\pm 95 \%$ confidence intervals) of blue-green coloration (R[400-575 nm]/R[300-700 nm]) of eggs laid (1) by females with experimentally removed wing feathers (experimental), (2) by females with sham-manipulated feathers, and (3) by control females. Sample sizes for each group are shown.

males (treatment effect: $F=2.98, \mathrm{df}=2,29, P=.067$; post hoc comparisons, least significant difference (LSD) tests: experimental vs. control: $P=.049$; experimental vs. sham manipulated: $P=.040$ ). Moreover, since blue-green color intensity of eggs laid by females in the two control groups did not vary (post hoc comparison, LSD test: control vs. sham manipulated: $P=.81$ ), we pooled data from the two control groups and increased statistical power of the comparison of egg coloration between control and experimental females $(F=6.08, \mathrm{df}=1,30, P=.020)$. This result did not change qualitatively even after including in the control group females that were not captured before laying ( $F=6.19$, df $=1,37, P=.018$; fig. 3 ). Furthermore, our feather removal experiment did not affect clutch size (treatment effect: $F=1.30, \mathrm{df}=2,29, P=$ .29; post hoc comparisons, LSD tests: $P \geq .12$ ) or egg size (treatment effect: $F=0.02, \mathrm{df}=2,29, P=.98$; post hoc comparisons, LSD tests: $P \geq .83$ ). All these results together suggest that egg color was affected by the experimental manipulation of female body condition.

\section{Egg Color and Parental Provisioning}

Provisioning effort of spotless starling males in nests where eggs were not removed (mean feeding rate per hour \pm $\mathrm{SE}=1.94 \pm 0.24)$ did not differ significantly from that recorded in experimental nests (mean feeding rate per hour $\pm \mathrm{SE}=1.57 \pm 0.23 ; F=1.40, \mathrm{df}=1,70.8, P=$ .24) after taking into account the effect of nestling development stage $(F=32.7, \mathrm{df}=2,69, P<.0001)$ and statistically controlling for number of nestlings $(F=0.004$, $\mathrm{df}=1,69, P>.9)$, hatching date $(F=1.66, \mathrm{df}=1,69$, $P=.2)$, provisioning rate of females $(F=0.46, \mathrm{df}=$ $1,69, P=.5$ ), and variation in nestling age within each nestling development stage $(F=0.72$, df $=1,69, P=$ .40). This conclusion did not vary after removing from the model all nonsignificant terms (effect of the experiment; $F=0.14$, df $=1,50.5, P>.7)$. Therefore, provisioning rates detected in experimental nests (i.e., those that incubated artificial model eggs) are within the natural variation in provisioning effort of the population.

Blue-green egg chroma of natural eggs was not significantly related to female feeding effort (first nestling development stage: $r=0.13, N=44, P=.4$; second stage: $r=0.08, N=40, P=.6$; third stage: $r=-0.01, N=$ 34, $P=.96)$, clutch size $(r=-0.06, N=44, P=.7)$, or egg size $(r=-0.25, N=42, P=.1)$. However, males from nests with experimental dark eggs fed the nestlings more frequently than those from nests with experimental pale eggs (fig. 4; final model: $F=7.75$, df $=1,44.4$, $P=.008$; full model: $F=4.10, \mathrm{df}=1,49.7, P=.048$ ). Number of nestlings in the nest (full model: $F=0.02$, $\mathrm{df}=1,30, P>.9)$, variation in nestling age within each nestling development stage (full model: $F=0.32, \mathrm{df}=$ $1,30, P=.58$ ), and female provisioning effort (full model: $F=1.56, \mathrm{df}=1,30, P=.22)$ did not explain a significant proportion of the variance in male provisioning rate. Apart from experimental treatment (see above), only nestling development stage ( $F=7.83, \mathrm{df}=2,35, P=.0015)$, nest identity $(F=2.67$, df $=20,35, P=.005)$, and hatching date (final model: $F=3.17, \mathrm{df}=1,35, P=$ .08) remained in the final model. Although females tended to feed their nestlings more frequently in nests with ex-

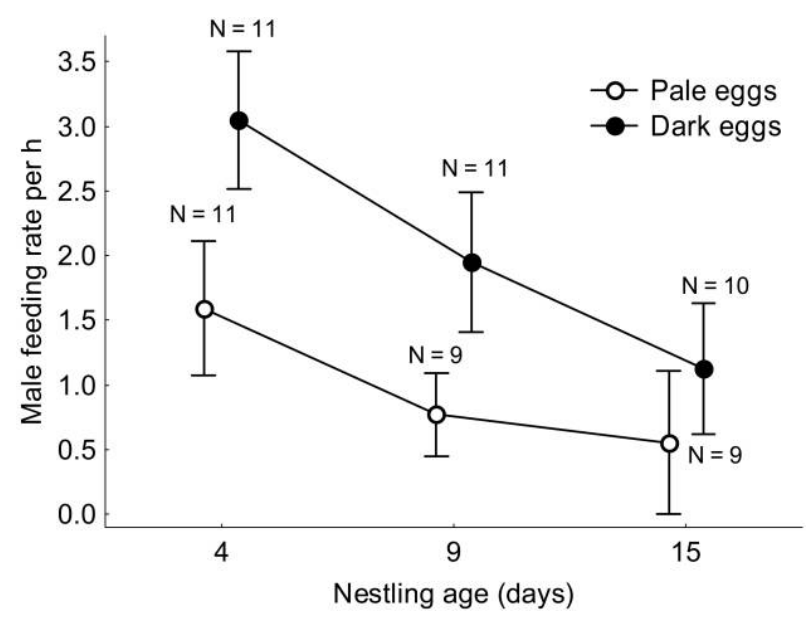

Figure 4: Mean number ( \pm SE) of provisioning visits per hour performed by males in experimental nests with pale or dark blue-greenish eggs at three different stages of nestling development. 


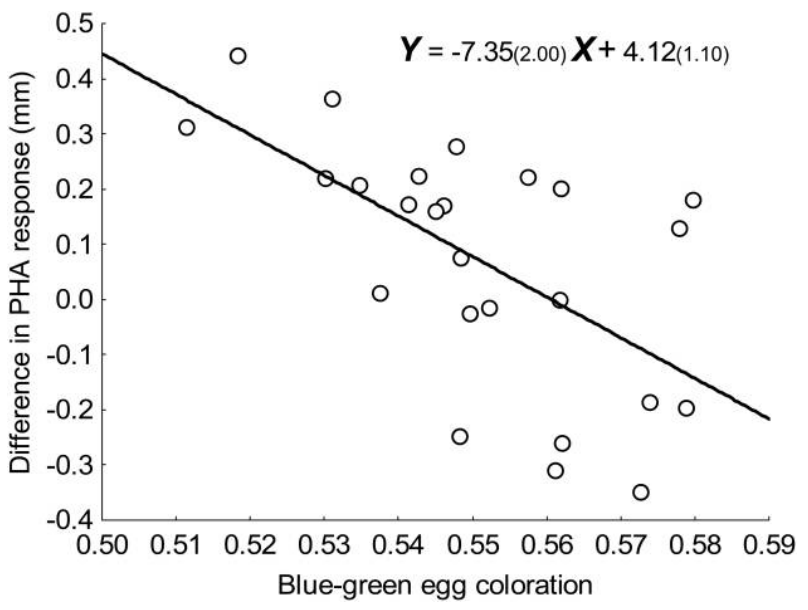

Figure 5: Relationship between blue-green coloration (R[400-575 nm]/ $\mathrm{R}[300-700 \mathrm{~nm}]$ ) of eggs and difference in PHA response of foodsupplemented and control nestlings of the same nest. Regression equation (SE) is also shown.

perimental dark eggs (mean feeding rate per hour \pm $\mathrm{SE}=5.35 \pm 0.32$ vs. $4.12 \pm 0.47$ ), differences did not reach statistical significance $(F=3.62, \mathrm{df}=1,45.8$, $P=.06)$ after controlling for the effect of male feeding effort $(F=0.47, \mathrm{df}=1,36, P=.50)$, laying date $(F=$ 2.93, $\mathrm{df}=1,36, P=.096)$, variation in nestling age within each nestling development stage $(F=3.01, \mathrm{df}=$ $1,36, P=.091)$, observation time $(F=0.94, \mathrm{df}=1,36$, $P=.40)$, and nest identity $(F=3.08, \mathrm{df}=20,36, P=$ .002 ). As a consequence, total number of feeding bouts was larger in nests with dark eggs (mean feeding rate per hour $\pm \mathrm{SE}=5.05 \pm 0.53)$ than in nests with pale eggs (mean feeding rate per hour $\pm \mathrm{SE}=7.36 \pm 0.51$; full model, treatment effect: $F=10.10, \mathrm{df}=1,53, P=.002$ ).

\section{Egg Color and Nutritional Conditioned Traits of Nestlings}

As predicted, the effect size of our food supply experiment on PHA response of nestlings was larger in nests with eggs reflecting less at the blue-green wavelength (R[400-575]/ $\mathrm{R}[300-700]: r=-0.61, N=25, P=.001$; fig. 5). The statistical significance of this relationship did not depend on the negative effect of the food supply experiment on PHA response detected in some nests (i.e., PHA response of control nestlings resulting larger than that of experimental nestlings) because when only using nests with positive effects, the relationship is still negative and significant $(r=-0.53, N=17, P=.029)$. Moreover, laying date (partial $\beta \pm \mathrm{SE}=0.358 \pm 0.151, \quad t=2.37, \quad \mathrm{df}=22$, $P=.027)$, but not clutch size $(P=.8)$ or number of nestlings $(P=.8)$, explained a significant portion of the variance in difference of PHA response when added to the model, in addition to the variance explained by the intensity of blue-green egg color (partial $\beta \pm \mathrm{SE}=$ $-0.610 \pm 0.151, t=4.04, \mathrm{df}=22, P=.0006)$. Therefore, the effect of the experimental food supply varied depending on blue-green color intensity of the eggs. Finally, we also detected that the relationship between PHA response and egg coloration differed between experimental and control nestlings (repeated-measured ANCOVA, PHA response as dependent variable; experimental treatment as a within factor: $F=13.9, \mathrm{df}=1,23, P=.0011$; interaction between intensity of blue-green color and experimental treatment: $F=13.5, \quad \mathrm{df}=1,23, \quad P=.0013)$. While PHA response in nests with low-colored eggs tended to be lower for control nestlings than for experimental ones, the opposite occurred in nests with eggs reflecting more at the blue-green wavelength (fig. 6).

\section{Discussion}

In this study, we have found several lines of evidence supporting the hypothesis that blue-green color intensity of spotless starling eggs is a sexually selected trait of females that affects paternal feeding effort (SSH). We discuss the experimental methodologies used, the results in the context of the SSH, and also other alternative hypotheses explaining our results.

The experiment of wing feather clipping, which invariably provokes high energy expenditure associated with flying activities (Møller et al. 1995), resulted in a larger rate of body mass loss of experimental in comparison with control spotless starling females (see "Results"). This ex-

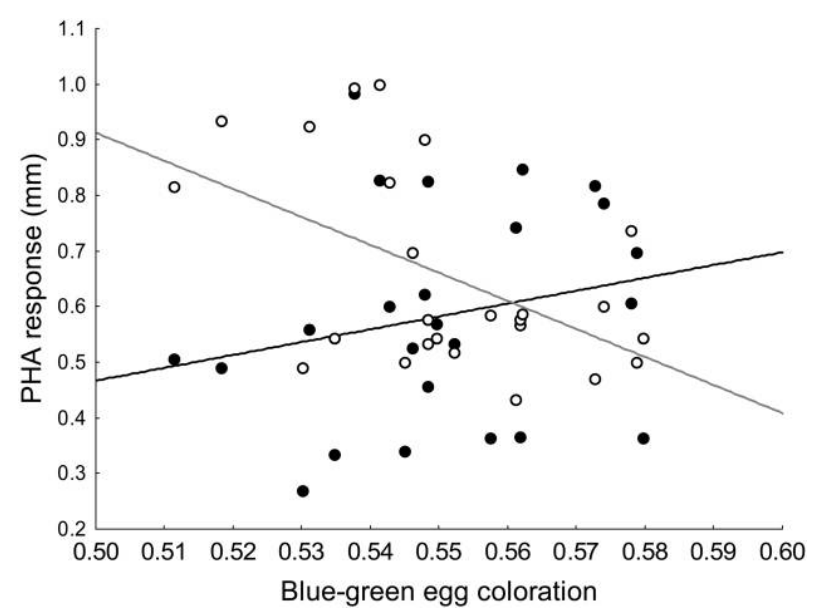

Figure 6: Relationship between blue-green coloration (R[400-575 nm]/ $\mathrm{R}[300-700 \mathrm{~nm}]$ ) of eggs and PHA response of food-supplemented (open circles, gray line) and control (filled circles, black line) nestlings. 
perimental effect was detected before egg laying, and thus experimental females were in poorer condition than control females when producing the eggshell. Moreno and Osorno (2003) predicted a relationship between female condition and intensity of blue-green egg coloration because it should reflect levels of oxidative stress in laying females. The use of biliverdin in the eggshell (i.e., bluegreen color) would reflect the oxidative condition of females because this pigment is a potent antioxidant. In accordance with the prediction, a positive relationship between body condition of females and intensity of bluegreen color of their eggs has been empirically shown in different species (see the first section of this article). Furthermore, Moreno et al. (2006a) showed in a food supplementation experiment that pied flycatcher females provided with supplementary food before and during laying laid more intensely colored blue-green eggs than did control females. In this study, we did not manipulate body condition of females by food supplementation but by removal of wing feathers, which provoked an extra effort in flying activities. The effect of this experiment on the intensity of eggshell coloration was predicted solely by the SSH because of its effect on female oxidative condition. Because we did not manipulate female nutritional condition directly, differences in egg coloration between experimental and control females cannot be explained by a direct effect of pigments in the diet. Consequently, the effect of wing feather removal on egg color intensity is consistent with the hypothesis that egg color intensity signals phenotypic condition in spotless starling females.

Our second experiment investigated the effect of egg color per se on paternal feeding effort. As predicted by other postmating sexually selected characters (Burley 1988; Sheldon 2000), because intense blue-green eggs in the spotless starling indicate females in good body condition, males paired with such females should invest differentially in reproduction (Moreno and Osorno 2003). However, to know whether egg color is directly affecting male provisioning effort, the association between egg color and female condition needs to be broken, because males might be using other clues instead of egg color to assess female condition. We managed to do this by using experimental model eggs. These artificial eggs do not reflect at the UV wavelengths, but their blue-green chroma was within the limits of blue-green coloration in natural eggs. Differences between experimental and natural eggs in UV chroma did not result in different feeding rates of males attending nests with natural and experimental eggs (see "Results"). Moreover, at the visual part of the spectrum $(400-700 \mathrm{~nm})$, reflectance curves of both dark and pale experimental model eggs showed a shape similar to the curve estimated for natural eggs (fig. 1), and chroma of experimental model eggs was within the natural egg variation (fig. 2). Thus, since pale and dark experimental model eggs greatly differed in their blue-green chroma, our experimental approach was appropriate to test the effect of egg coloration on male feeding effort.

After controlling for some potential confounding variables, including female provisioning rate, we found that males with intense blue-green artificial eggs in their nests fed the nestlings more frequently than did those males with pale artificial eggs (fig. 4). An association between natural egg color and male provisioning effort cannot explain this result because experimental treatments were randomly assigned and natural eggs were removed from the nests soon after laying (within a few hours). Moreover, nestlings raised in experimental nests were not related to adults feeding them, and thus the possible relationship between natural egg coloration and nestling phenotypic quality (e.g., immunocompetence; see above) was broken with our experimental design. We are not aware of any hypothesis that could explain the effect of the experiment with model eggs on paternal feeding effort apart from the $\mathrm{SSH}$. Therefore, experimental evidence supporting the predicted relationships between egg coloration and paternal feeding effort and between female condition and egg coloration strongly suggests that blue-green intensity of eggs is a sexually selected signal in spotless starling females that affects parental effort of their mates (Moreno and Osorno 2003).

Experimental support for the predicted relationship between egg color and paternal feeding effort was recently found in pied flycatchers but not in collared flycatchers. Moreno et al. (2006b) detected that egg coloration of crossfostered eggs predicted relative male contribution to nestling provisioning in pied flycatchers. However, Krist and Grim (2007) did not find such a relationship when studying collared flycatcher nests with cross-fostered eggs. In these two experiments, however, males fed nestlings that hatched from the same eggs that males had seen in their nests. There is evidence of a relationship between egg color and differential maternal investment in eggs (e.g., antibodies; Morales et al. 2006), and this maternal investment could affect the phenotypic quality of nestlings (e.g., immunocompetence). Therefore, the results found in pied flycatchers might be also explained as a consequence of males adjusting their provisioning effort to offspring phenotypic quality rather than to egg color.

The hypothesis of egg coloration being a postmating sexually selected signal in females also predicts better environmental conditions for nestling growth in nests with intense blue-green eggs. In our experiment with model eggs, we detected an effect of egg coloration on paternal feeding effort. Moreover, females also tended to feed their nestlings more frequently in nests with artificial dark eggs. The effect of the experiment on female feeding effort, al- 
though not statistically significant, may suggest that females used the feeding effort of their mates to adjust their own feeding effort. In accordance with this possibility, we found that female and male feeding effort were significantly related ( $r=0.52, N=20, P=.019)$ but only for the first nestling development stage. In any case, although our results suggest a better nutritional environment for nestlings reared in nests where artificial dark eggs had been incubated, we did not find any significant effect of the experiment on nestling phenotypic quality measured at the end of the nesting period (i.e., PHA response, body mass, and body condition; comparisons between nestlings reared in nests with experimental pale and dark eggs: $t \leq 1.08$, df $=16, P \geq .29$ ). However, sample sizes were quite small (see fig. 3), and thus Type II error (the risk of not rejecting the null hypothesis when it is false) was quite large. In addition, we reduced brood size of all experimental nests to two or three nestlings, partly to obtain nests with similar brood size and partly also to reduce nestling mortality due to brood reduction. The low level of sibling competition in experimentally reduced broods would make it difficult to find significant differences in nestling phenotypic condition between nests with pale and dark eggs. Given all these potential problems, to test the prediction that blue-green egg color intensity in the spotless starling predicts environmental (i.e., nutritional) conditions that nestlings experience during development, we performed a food supply experiment in a subsample of nests. In agreement with previous food supply experiments performed in other passerines (e.g., magpies: De Neve et al. 2004b, 2004c; barn swallows: Saino et al. 1997), we found that PHA response (but not body size or condition) of nestlings was affected by the experiment. Since we manipulated nutritional conditions of some nestlings in the nest, between-nest variation in the effect size of our experiment (i.e., difference in PHA response between experimental and control nestlings) should reflect betweennest variation in nutritional environments that nestlings (both experimental and control ones) experienced during growth. Therefore, we used the difference in levels of immune response between experimental and control nestlings in the same nest as an index of nutritional conditions experienced by nestlings. In accordance with this assumption, we found that laying date was positively related to the difference in PHA response between experimental and control siblings (see "Results"). Laying date is a life-history variable generally negatively related to other variables reflecting nestling phenotypic quality. This relationship is usually explained as the result of a decrease in resource availability as the season progresses (timing hypothesis) and also the result of genetic and/or phenotypic quality of breeders (i.e., parental quality) may decrease with time (quality hypothesis; e.g., Verhulst et al. 1995; Verboven and Verhulst 1996; Verboven and Visser 1998; De Neve et al. 2004a). Both hypotheses suggest that the relationship between laying date and nestling phenotypic quality is mediated by nutritional conditions that nestlings experience during development. Therefore, the fact that the effect of our experimental food supply increased as the season progressed supports our assumption that differences in PHA response can be interpreted as indicators of nestling nutritional conditions.

As predicted by the SSH, our experiment of food supplementation provoked stronger positive effects in nests where eggs reflected less at the blue-green wavelengths (400-575 nm; fig. 5). Furthermore, the statistical significance of such a relationship did not depend on the influence of some nests where the immune response was lower in food-supplemented nestlings than in control ones (see "Results"). Since we did not record parental feeding behavior in these experimental nests, we cannot establish a direct link between our results and paternal feeding effort. An alternative explanation would be that egg coloration and offspring quality (i.e., immunocompetence) were positively related through maternal effects or genetic traits of females that correlate with egg coloration (Morales et al. 2006). However, even if that was the case, a differential investment of males in highly colored clutches would have a selective advantage (see Sheldon 2000). Results from our egg color experiment showed a differential reproductive effort of males that encountered experimental dark eggs; therefore, the finding that blue-green color intensity predicted the effect of experimental food supply on PHA response of nestlings can be interpreted as being consistent with the hypothesis that egg coloration is a sexually selected signal in females.

For nests with very blue-green eggs, our food supplementation experiment tended to have negative effects, with experimental nestlings showing lower immune response than their control siblings (see fig. 5). This result was mainly due to the negative relationship between PHA response and blue-green coloration detected for experimental but not for control nestlings (fig. 6) and may be conciliated with the hypothesis of blue-green coloration predicting nutritional conditions experienced by nestlings during development. For instance, it is likely that our food supplementation affected nestling begging behavior by decreasing nestling solicitation. Consequently, experimental nestlings would have been fed by parents at a lower frequency than expected in the absence of experimental feeding. Moreover, if experimental nestlings demanded less food, the parents might have allocated more food to the rest of the brood. Thus, our experiment may have affected the nutritional condition of both experimental and control nestlings. Interestingly, the SSH also predicts that parents should provide not only a larger quantity but also a higher 
quality of food for their offspring in nests with more bluegreen eggs than in nests with paler eggs (see Moreno and Osorno 2003). Therefore, on the one hand, it is possible that our experimental food supplementation provoked satiation in experimental nestlings, and control nestlings received a larger proportion of the food carried to the nest by parents. On the other hand, it is also possible that parents in nests with more colored eggs provided food of higher quality for their nestlings, contributing to a larger improvement of the food received by control nestlings in this kind of nest. If these assumptions are met, a negative effect of our food supplementation on nutritional conditioned traits (e.g., PHA response) in nests with very bluegreen eggs would be plausible. However, we have no data on begging behavior of experimental and control nestlings or on quality of the food provided by adults, and hence this scenario is merely speculative.

Female sexual ornaments have been traditionally neglected in the study of sexual selection (Amundsen 2000). Most female ornaments have been studied in relation to mating investment (Amundsen and Forsgren 2001), but they are almost unexplored in a postmating sexual selection context (but see Pilastro et al. 2003). The hypothesis of egg coloration being a postmating sexually selected trait offers the possibility of studying processes of sexual selection in females. Until now, research efforts have been concentrated on studying predictions of the hypothesis and finding empirical, comparative, and experimental support (see the first section of this article). In this study, we found experimental support for the main predictions of the hypothesis in spotless starlings, and it constitutes one of the few experimental evidences of differential allocation in male parental investment in relation to a female character. Although more experiments to validate the hypothesis in other species are necessary, we think it is time to further explore quality signaling in females, assuming that the blue-green color of the eggs in many species is, at least in part, the result of postmating sexual selection.

\section{Acknowledgments}

This research was funded by the Spanish Ministry of Education and Science and the Fondo Europeo de Desarrollo Regional (FEDER; CGL2004-01777/BOS). The authorization for carrying out the experiments was granted by Dirección General de Gestión del Medio Natural, Consejería de Medio Ambiente, Junta de Andalucía. We thank C. Zamora and three anonymous reviewers for their comments and suggestions on a previous version of the manuscript.

\section{Literature Cited}

Alonso-Alvarez, C., and J. L. Tella. 2001. Effects of experimental food restriction and body-mass changes on the avian T-cell-mediated immune response. Canadian Journal of Zoology 79:101-105.

Alonso-Alvarez, C., S. Bertrand, G. Devevey, J. Prost, B. Faivre, and G. Sorci. 2004. Increased susceptibility to oxidative stress as a proximate cost of reproduction. Ecology Letters 7:363-368.

Amundsen, T. 2000. Why are female birds ornamented? Trends in Ecology \& Evolution 15:149-155.

Amundsen, T., and E. Forsgren. 2001. Male mate choice selects for female coloration in a fish. Proceedings of the National Academy of Sciences of the USA 98:13155-13160.

Aviles, J. M., J. J. Soler, and T. Perez-Contreras. 2006a. Dark nests and egg colour in birds: a possible functional role of ultraviolet reflectance in egg detectability. Proceedings of the Royal Society B: Biological Sciences 273:2821-2829.

Aviles, J. M., J. J. Soler, T. Perez-Contreras, M. Soler, and A. P. Møller. $2006 b$. Ultraviolet reflectance of great spotted cuckoo eggs and egg discrimination by magpies. Behavioral Ecology 17:310-314.

Burley, N. 1986. Sexual selection for aesthetic traits in species with biparental care. American Naturalist 127:415-445.

- 1988. The differential-allocation hypothesis: an experimental test. American Naturalist 132:611-628.

Cramp, S. 1998. Cramp's the complete birds of the Western Palearctic. Optimedia, Oxford University Press, Oxford.

Davies, N. B. 2000. Cuckoos, cowbirds, and others cheats. Poyser, London.

Davies, N. B., and M. de L. Brooke. 1988. Cuckoos versus reed warblers: adaptations and counteradaptations. Animal Behaviour 36:262-284.

De Neve, L., J. J. Soler, M. Soler, and T. Perez-Contreras. 2004a. Differential maternal investment counteracts for late breeding in magpies Pica pica: an experimental study. Journal of Avian Biology 35:237-245.

De Neve, L., J. J. Soler, M. Soler, T. Perez-Contreras, M. MartinVivaldi, and J. G. Martinez. 2004b. Effects of a food supplementation experiment on reproductive investment and a post-mating sexually selected trait in magpies Pica pica. Journal of Avian Biology 35:246-251.

De Neve, L., J. J. Soler, M. Soler, and T. Perez-Contreras. 2004c. Nest size predicts the effect of food supplementation to magpie nestlings on their immunocompetence: an experimental test of nest size indicating parental ability. Behavioral Ecology 15:1031-1036.

Falchuk, K. H., J. M. Contin, T. S. Dziedzic, Z. Feng, T. C. French, G. J. Heffron, and M. Montorzi. 2002. A role for biliverdin IX $\alpha$ in dorsal axis development of Xenopus laevis embryos. Proceedings of the National Academy of Sciences of the USA 99:251-256.

Gosler, A. G., J. P. Higham, and S. J. Reynolds. 2005. Why are birds' eggs speckled? Ecology Letters 8:1105-1113.

Goto, N., H. Kodama, K. Okada, and Y. Fujimoto. 1978. Suppression of phytohemagglutinin skin response in thymectomized chickens. Poultry Science 57:246-250.

Kennedy, G. Y., and H. G. Vevers. 1976. A survey of avian eggshell pigments. Comparative Biochemistry and Physiology B 55:117123.

Kennedy, M. W., and R. G. Nager. 2006. The perils and prospects of using phytohaemagglutinin in evolutionary ecology. Trends in Ecology \& Evolution 21:653-655. 
Kilner, R. M. 2006. The evolution of egg colour and patterning in birds. Biological Reviews 81:383-406.

Krist, M., and T. Grim. 2007. Are blue eggs a sexually selected signal of female collared flycatchers? a cross-fostering experiment. Behavioral Ecology and Sociobiology 61:863-876.

Martin, L. B., P. Han, J. Lewittes, J. R. Kuhlman, K. C. Klasing, and M. Wikelski. 2006. Phytohemagglutinin-induced skin swelling in birds: histological support for a classic immunoecological technique. Functional Ecology 20:290-299.

Møller, A. P., M. Lindén, J. J. Soler, M. Soler, and J. Moreno. 1995. Morphological adaptations to an extreme sexual display, stonecarrying in the black wheatear, Oenanthe leucura. Behavioral Ecology 6:368-375.

Morales, J., J. J. Sanz, and J. Moreno. 2006. Egg colour reflects the amount of yolk maternal antibodies and fledging success in a songbird. Biology Letters 2:334-336.

Moreno, J., and J. L. Osorno. 2003. Avian egg colour and sexual selection: does eggshell pigmentation reflect female condition and genetic quality? Ecology Letters 6:803-806.

Moreno, J., J. L. Osorno, J. Morales, S. Merino, and G. Tomas. 2004. Egg colouration and male parental effort in the pied flycatcher Ficedula hypoleuca. Journal of Avian Biology 35:300-304.

Moreno, J., J. Morales, E. Lobato, S. Merino, G. Tomas, and J. Martinez-de la Puente. 2005. Evidence for the signaling function of egg color in the pied flycatcher Ficedula hypoleuca. Behavioral Ecology 16:931-937.

Moreno, J., E. Lobato, J. Morales, S. Merino, G. Tomas, J. Martinezde la Puente, J. J. Sanz, R. Mateo, and J. J. Soler. 2006a. Experimental evidence that egg color indicates female condition at laying in a songbird. Behavioral Ecology 17:651-655.

Moreno, J., J. Morales, E. Lobato, S. Merino, G. Tomas, and J. Martinez-de la Puente. 2006b. More colourful eggs induce a higher relative paternal investment in the pied flycatcher Ficedula hypoleuca: a cross-fostering experiment. Journal of Avian Biology 37: $555-560$.

Motis, A., J. Estrada, and D. Oro. 1997. Nestling diet of the spotless starling Sturnus unicolor and the European starling Sturnus vulgaris in a sympatric breeding area. Ornis Fennica 74:179-185.

Petrie, M., and A. P. Møller. 1991. Laying eggs in others' nests: intraspecific brood parasitism in birds. Trends in Ecology \& Evolution 6:315-320.

Pilastro, A., M. Griggio, and G. Matessi. 2003. Male rock sparrows adjust their breeding strategy according to female ornamentation: parental or mating investment? Animal Behaviour 66:265-271.

Saino, N., S. Calza, and A. P. Møller. 1997. Immunocompetence of nestling barn swallows in relation to brood size and parental effort. Journal of Animal Ecology 66:827-836.

Schulte-Hostedde, A. I., B. Zinner, J. S. Millar, and G. J. Hickling. 2005. Restitution of mass-size residuals: validating body condition indices. Ecology 86:155-163.

Sheldon, B. C. 2000. Differential allocation: tests, mechanisms and implications. Trends in Ecology \& Evolution 15:397-402.
Siefferman, L., K. Navara, and G. Hill. 2006. Egg coloration is correlated with female condition in eastern bluebirds (Sialia sialis). Behavioral Ecology and Sociobiology 59:651-656.

Soler, J. J., and A. P. Møller. 1996. A comparative analysis of the evolution of variation in appearance of eggs of European passerines in relation to brood parasitism. Behavioral Ecology 7:89-94.

Soler, J. J., and M. Soler. 2000. Brood-parasite interactions between great spotted cuckoos and magpies: a model system for studying coevolutionary relationships. Oecologia (Berlin) 125:309-320.

Soler, J. J., J. Moreno, J. M. Avilés, and A. P. Møller. 2005. Blue and green egg-color intensity is associated with parental effort and mating system in passerines: support for the sexual selection hypothesis. Evolution 59:636-644.

Solis, J. C., and F. de Lope. 1995. Nest and egg crypsis in the groundnesting stone curlew Burhinus oedicnemus. Journal of Avian Biology 26:135-138.

StatSoft. 2001. STATISTICA for Windows (computer program manual). Version 6.0. StatSoft, Tulsa, OK.

Stocker, R., Y. Yamamoto, A. F. McDonagh, A. N. Glazer, and B. N. Ames. 1987. Bilirubin is an antioxidant of possible physiological importance. Science 235:1043-1046.

Stokke, B. G., A. Moksnes, and E. Røskaft. 2002. Obligate brood parasites as selective agents for evolution of egg appearance in passerine birds. Evolution 56:199-205.

Underwood, T. J., and S. G. Sealy. 2002. Adaptive significance of egg coloration. Pages 280-289 in D. C. Deeming, ed. Avian incubation, behaviour, environment and evolution. Oxford University Press, Oxford.

Veiga, J. P., J. Moreno, P. J. Cordero, and E. Mínguez. 2001. Territory size and polygyny in the spotless starling: resource-holding potential or social inertia? Canadian Journal of Zoology 79:19511956.

Veiga, J. P., J. Moreno, M. Arenas, and S. Sanchez. 2002. Reproductive consequences for males of paternal vs. territorial strategies in the polygynous spotless starling under variable ecological and social conditions. Behaviour 139:677-693.

Verboven, N., and S. Verhulst. 1996. Seasonal variation in the incidence of double broods: the date hypothesis fits better than the quality hypothesis. Journal of Animal Ecology 65:264-273.

Verboven, N., and M. E. Visser. 1998. Seasonal variation in local recruitment of great tits: the importance of being early. Oikos 81: 511-524.

Verhulst, S., J. H. Vanbalen, and J. M. Tinbergen. 1995. Seasonal decline in reproductive success of the great tit: variation in time or quality? Ecology 76:2392-2403.

Von Schantz, T., S. Bensch, M. Grahn, D. Hasselquist, and H. Wittzell. 1999. Good genes, oxidative stress and condition-dependent sexual signals. Proceedings of the Royal Society B: Biological Sciences 266:1-12.

Associate Editor: Ben C. Sheldon Editor: Michael C. Whitlock 